\title{
Una aproximación a la realidad de los centros públicos rurales en Andalucía
}

\author{
An approach to the reality of rural public schools in Andalusia
}

\author{
Miguel Pérez Ferra \\ e-mail:mperez@ujaen.es \\ Universidad de Jaén. España \\ Inmaculada García Martínez (iD \\ e-mail: igmartinez@ugr.es \\ Universidad de Granada. España \\ Rocío Quijano López \\ e-mail: rquijano@ujaen.es \\ Universidad de Jaén. España
}

\section{Resumen}

Se aborda la situación actual de las escuelas rurales analizando la legislación específica en Andalucía; valorando la realidad organizativo-didáctica que demandan y la situación del profesorado que accede a las mismas: permanencia efímera, así como formación inicial y continua no adecuadas. Las conclusiones evidencian que los centros rurales se resisten a sustituir el sentido de acogida de la comunidad educativa, por la prevalencia del rendimiento de cuentas, que deja a un lado la "integridad" del ser humano, determinante de la "integralidad" de los elementos constitutivos de la persona, así como la ausencia de arraigo de los maestros a esas realidades culturales.

Palabras clave: escuela rural; centros públicos; educación primaria; agrupamiento multigrado; Andalucía.

\section{Abstract}

The current situation of rural schools is analyzed by analyzing the specific legislation in Andalusia; valuing the organizational-didactic reality that they demand and the situation of the teaching staff that accesses them: ephemeral permanence, as well as inadequate initial and continuous training. The conclusions show that rural centers are reluctant to replace the sense of welcome of the educational community, because of the prevalence of accountability, which leaves aside the "integrity" of the human being, determinant of the "integrality" of the elements constitutive of the person, as well as the lack of roots of teachers to these cultural realities.

Keywords: rural schools; public schools; primary education; multigrade groups; Andalusia.

Recibido / Received: 08-09-2017

Aceptado / Accepted: 22-02-2018

Cómo referenciar este artículo / How to reference this article:

Pérez, M., García, I., \& Quijano, R. (2018). Una aproximación a la realidad de los centros públicos rurales en Andalucía. Tendencias Pedagógicas, 32, 147-160. doi: 10.15366/ tp2018.32.011 


\section{Introducción}

El panorama educativo español delinea varias modalidades de centros, en función a su ubicación, características demográficas, perfil del alumnado y nivel ISEC (Índice de Nivel Sociocultural). Desde la perspectiva legislativa, es cierto que gran parte de la normativa regula las particularidades de cómo deben ser los centros urbanos; sin embargo, no son extrapolables algunos aspectos de la norma al contexto rural. Las peculiaridades que presentan el contexto rural y una escasa investigación sobre este tipo de centros, plantean una creciente necesidad de profundizar en la realidad de los mismos, considerando la singularidad que confieren los diferentes contextos en los que se ubican.

Lo cierto es que hay una multiplicidad de connotaciones antropológicas que definen una gran diversidad de contextos y realidades, que diferencian unas escuelas rurales de otras, realidades que demandan intervenciones diferenciadas, tanto en el ámbito organizativo, didáctico-curricular, como en la relación con las familias y el entorno social. A groso modo vemos que, en el ámbito rural, el profesorado aborda situaciones para las que, normalmente, no ha sido preparado; desconoce el medio; el alumnado suele asociarse en grupos heterogéneos, de modo que las funciones del profesorado y del alumnado de más edad, adquieren otras dimensiones, que la organización escolar no suele contemplar. Respecto a las familias, indicar que las posibilidades de participación son mayores; sin embargo, no son reales, porque los padres ven la escuela como algo que no les atañe y necesitan del concurso del maestro o maestra para que se sientan útiles (Elboj, Gil, Rodrigo y Zaldívar, 2004).

\section{Metodología}

El panorama educativo español delinea varias modalidades de centros, en función a su ubicación, características demográficas, perfil del alumnado y nivel ISEC (Índice de Nivel Sociocultural). Desde la perspectiva legislativa, es cierto que gran parte de la normativa regula las particularidades de cómo deben ser los centros urbanos; sin embargo, no son extrapolables algunos aspectos de la norma al contexto rural. Las peculiaridades que presentan el contexto rural y una escasa investigación sobre este tipo de centros, plantean una creciente necesidad de profundizar en la realidad de los mismos, considerando la singularidad que confieren los diferentes contextos en los que se ubican.

\subsection{Objetivos de la investigación}

- Realizar una revisión legislativa sobre las necesidades que determinaron la nueva configuración organizativa de las escuelas rurales en Andalucía.

- Definir las características de los centros multigrado.

- Determinar las necesidades formativas específicas del profesorado de escuelas rurales y su actitud en este tipo de centros.

\subsection{Procedimiento seguido en la revisión}

La revisión que presentamos en este artículo se adecúa a las directrices referentes a la realización de una revisión sistemática, recogidas en la declaración PRISMA (Preferred Reporting Items for Systematic reviews and Meta-Analyses) de Urrutia y Bonfill (2010). El presente trabajo tiene como principal objetivo conocer las características y prácticas docentes que se llevan a cabo en los centros multigrado. Para la búsqueda se consultó en las bases de datos Scopus y Google Scholar. El periodo de esta búsqueda se comprende entre los meses junio y julio 2017, empleándose palabras clave como "escuela rural", "centros públicos", "educación primaria" o "agrupamiento multigrado". A continuación, se definió el rango temporal de la publicación de dichos artículos, siendo desde 2007 a 2017. En esta franja temporal se encontraron 65 artículos. 
Figura 1

Diagrama de flijo

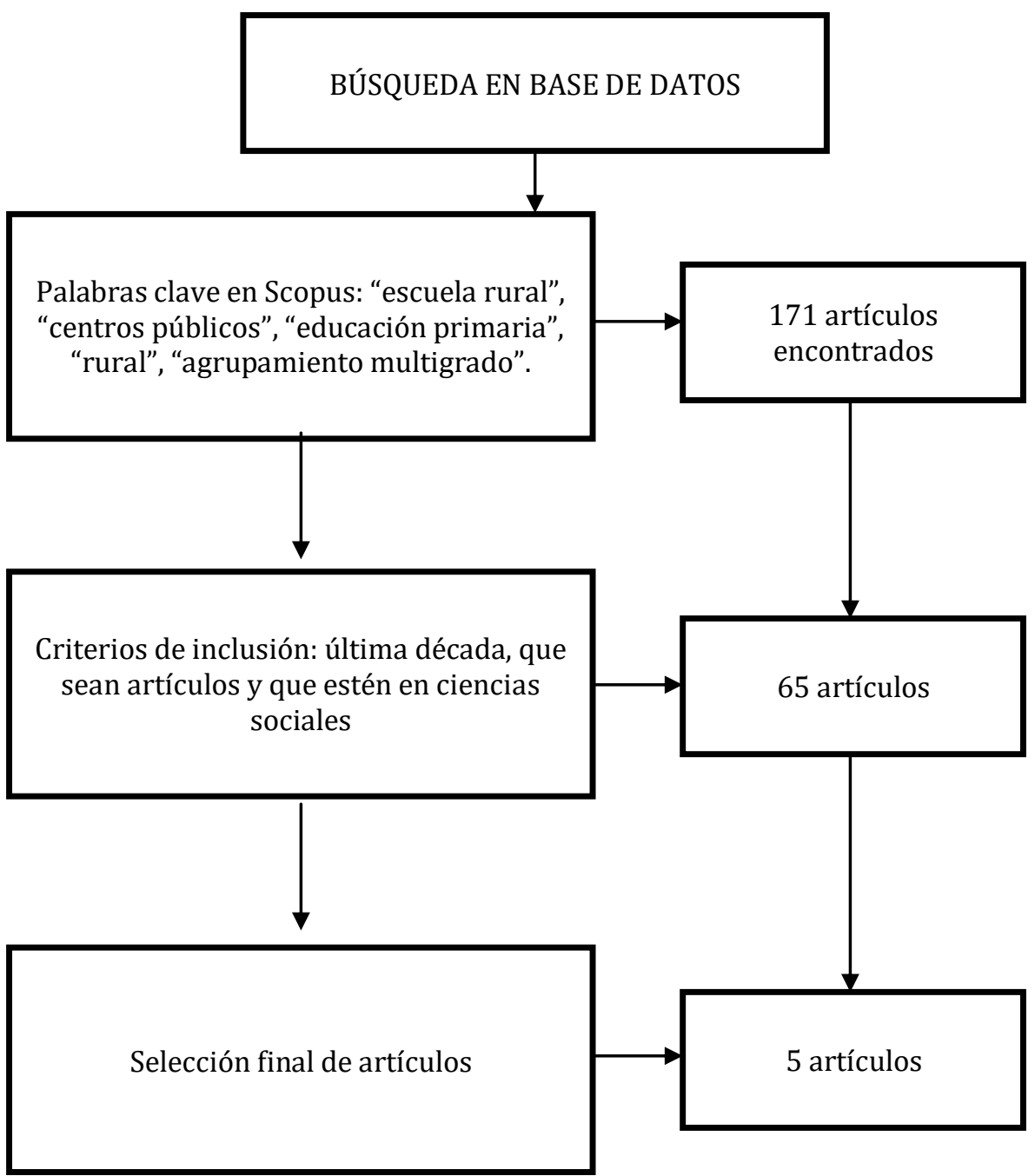

Fuente: elaboración propia.

Tabla 1

Artículos seleccionados

\begin{tabular}{|c|c|c|c|c|}
\hline Autor/es y año & $\begin{array}{l}\text { Tipo de } \\
\text { estudio }\end{array}$ & Población & Muestra & Instrumento \\
\hline $\begin{array}{l}\text { Block, Ramírez y Reséndiz } \\
\text { (2015) }\end{array}$ & $\mathrm{R}$ & Maestra & 1 & Estudio de caso \\
\hline Alvarado (2010) & $\mathrm{T}$ & $\begin{array}{l}\text { Estudiantes } \\
\text { mujeres }\end{array}$ & 12 & $\begin{array}{l}\text { Entrevistas, grupos } \\
\text { focales, observación } \\
\text { participante }\end{array}$ \\
\hline Bustos (2010) & $\mathrm{T}$ & $\begin{array}{l}\text { Maestros } \\
\text { primaria }\end{array}$ & 290 & $\begin{array}{l}\text { Cuestionario y estudio de } \\
\text { caso }\end{array}$ \\
\hline Bustos (2011) & $\mathrm{R}$ & - & - & - \\
\hline $\begin{array}{l}\text { Varela-Crespo, Varela- } \\
\text { Garrote y Lorenzo- } \\
\text { Castiñeiras (2016) }\end{array}$ & $\mathrm{T}$ & $\begin{array}{l}\text { Maestros } \\
\text { primaria }\end{array}$ & 2037 & Cuestionario \\
\hline
\end{tabular}

T: Transversal, L: Longitudinal, R: Revisión.

Fuente: elaboración propia. 


\section{Aproximación a la escuela rural, ¿¿qué son los centros multigrado?}

Hay diferencias acentuadas entre zonas urbanas y zonas rurales, determinadas por el tipo de actividad económica predominante, el estilo de vida, así como por la realidad cultural de las mismas. Considerando las diferencias aludidas, resulta necesario que las escuelas ejecuten acciones para satisfacer las necesidades de un alumnado con marcadas diferencias respecto al contexto ambiental urbano (Alvarado, 2010). En este ámbito surgen las escuelas rurales, cuyo profesorado, como se ha afirmado anteriormente, no siempre tiene la formación necesaria para intervenir en ese tipo de realidades formativas, dependiendo ello de la disposición personal de los docentes y de su buena voluntad para orientar el desarrollo de su identidad profesional. Las escuelas rurales han surgido como realidades educativas para grupos heterogéneos de alumnos, en función de diferentes niveles académicos y edades y con expectativas, inquietudes y necesidades formativas ligadas al medio rural en el que viven (Boix, 2004 y 2011).

Una mirada retrospectiva permite constatar como Andalucía ha sido una de las primeras comunidades autónomas que regularizó los agrupamientos escolares rurales y la segunda de España en número de centros rurales, después de Castilla-León. Pero, precisamente por ello, resulta controvertido el creciente desequilibrio entre el interés por determinar las funciones de los colegios públicos rurales al comienzo de su andadura y el desvanecimiento del interés mostrado por la Administración Pública Andaluza, respecto a la atención específica a estos centros, en cuanto al decurso de su funcionamiento (Bustos, 2007).

Para comprender el funcionamiento de las instituciones educativas de las zonas rurales andaluzas, es preciso destacar la escasa actualización en cuanto a materia legislativa desde el Decreto 29/1988, de 10 de febrero sobre constitución de colegios públicos rurales, hasta el Real Decreto 132/2010, de 12 de febrero, el cual regula los requisitos mínimos para que los centros impartan las enseñanzas del segundo ciclo de educación infantil, educación primaria y educación secundaria. Prueba de ello es la Resolución de 12 de septiembre de 2017, de la Dirección General de Profesorado y Gestión de Recursos Humanos de la Junta, por la que se determina el desarrollo de las líneas estratégicas de formación del profesorado (BOJA de 22 de septiembre), solo en la línea IV, 2.), se alude a: "La atención a los centros con entornos de aprendizaje específicos (compensación, rural, hospitalarios, centros específicos de EE, etc.), sin que se haya desarrollado el plan específico de ejecución, hasta la fecha.

El primero refleja la necesidad de compensación de las desigualdades socioeconómicas, culturales y demográficas que impiden el acceso a la educación o una disminución de la calidad de parte de la población educativa. Asimismo, se recogen una serie de medidas que refuerzan a las escuelas rurales por medio de la construcción de nuevos centros, mantenimiento y reapertura de escuelas situadas en zonas rurales, el desarrollo de programas de educación compensatoria, contextualizados al entorno rural, así como la elaboración de un "Plan de Actuación para la Escuela Rural en Andalucía". Para materializar estas cuestiones, entre otras medidas, se propuso el establecimiento de agrupaciones de escuelas rurales con aulas diseminadas, situadas en una misma zona o comarca o varias, que constituían un único centro. A su vez, en este decreto también se estableció la dotación de más recursos materiales y humanos, siempre y cuando las posibilidades presupuestarias lo permitiesen.

La capacitación y formación docente también es contemplada en el citado decreto. En el mismo, se hace un llamamiento a un régimen singular de provisión de plazas que permita la selección y continuidad del profesorado, además de establecer un plan específico de formación y perfeccionamiento docente.

Por su parte, el Real Decreto 132/2010, de 12 de febrero, apenas hace referencia a los Centros Públicos Rurales, dedicando solamente un párrafo donde se les dispensa de algunos requisitos mínimos a alcanzar, al tiempo que se señala su característica modalidad de agrupamiento, afirmando que su unidad escolar alude a: "la agrupación de alumnos atendidos conjunta y simultáneamente por un profesor de manera ordinaria, independientemente del nivel al que pertenezcan" (Disposición 
adicional tercera, punto 2).

Los Centros Públicos Rurales fueron una consecuencia de la transformación de los centros rurales a finales de los ochenta y principios de los noventa. Conviene recordar el Decreto 29/1988, BOJA de 4 de marzo sobre constitución de colegios públicos rurales en la Comunidad Autónoma Andaluza, que dio lugar al Plan de Actuación para la Escuela Rural en Andalucía. Lo significativo de ese plan fue establecer una distribución más adecuada de las escuelas, en función de las localidades que tienen las mismas necesidades.

La política enunciada dio respuestas a la necesidad de transformación escolar en este tipo de contextos, cuyos indicadores más significativos fueron el difícil acceso geográfico a muchas localidades y el escaso número de habitantes (Hinojo, Raso e Hinojo, 2010). Las escuelas rurales dejarían de existir únicamente en el caso de que el entorno rural no tuviese posibilidades de subsistir (Moyano, 2002). Con el fin de subsanar las posibles desventajas de este tipo de escuelas frente a las situadas en contextos urbanos, Corchón (2000) aboga por un modelo escolar "contextualizado" y aplicable a la realidad rural, que recogiera las necesidades comunes de las escuelas ubicadas en territorios con estas casuísticas.

En este sentido, se alude a un modelo que incluye una doble vertiente curricular: aplicación del modelo normalizado para el conjunto de escuelas rurales andaluzas que se agruparan y formasen, un Colegio Público Rural, y una actuación diferenciada para aquellas Escuelas Rurales de hasta cuatro unidades y que no pudieran agruparse y formar un CPR (la denominación de Escuela Rural no existe en la actualidad). Los centros que por su ubicación no pudiesen agruparse con otros serían independientes en el ámbito administrativo y jurídico, constituyéndose en un solo centro educativo, con un nombre único, un sólo Proyecto de Centro, Consejo Escolar, Claustro de Profesores y Equipo Directivo. La normativa limitaba los agrupamientos a cuatro unidades como máximo, con aulas diseminadas, situadas en una misma zona o comarca, de las mismas o distintas localidades, de forma que se constituyesen como un solo centro educativo.

Al mismo tiempo que se ha ido forjando un marco normativo, han aparecido investigaciones que han arrojado luz sobre este tipo de centros, a menudo desapercibidos. Así, parafraseando a Cantón (2004), se propone un conjunto de características para esta modalidad de escolarización, a saber:

- Baja o muy baja ratio alumno - profesor.

- La forma de agrupamiento suele ser multigrado.

- En algunos casos es el último servicio público que queda en la localidad.

- Tiene ciertas dificultades para acceder a los bienes culturales, dando lugar cierto aislamiento en su alumnado.

Autores como Bernal-Agudo (2009) han incidido en las peculiaridades que definen a la escuela rural. Considera el autor que tiende a ser más diversa que la urbana, atendiendo a parámetros demográficos, físicos, culturales y económicos en los que se ubique el centro. Debido a estas particularidades, no es de extrañar que el número de alumnos matriculados en este tipo de centros sea escaso, de ahí que se formen grupos multinivel con alumnado de diferentes edades y cursos. Las circunstancias aludidas presentan al profesorado que trabaja en estos centros un gran reto: dar respuesta a las necesidades de un alumnado diverso con distintos ritmos de aprendizaje, motivaciones, gustos, etc., que también suceden en las aulas ordinarias, pero, generalmente, con diferencias menos acentuadas A cambio, este tipo de contextos ofrecen una elevada tasa de participación en los alumnos y en el mismo entorno, lo que apoya y facilita considerablemente la tarea del maestro/a.

Definidas las características de los centros rurales, conviene plantear el perfil profesional del profesorado de la escuela rural, puntos fuertes y débiles de este tipo de escuelas, agrupamientos más comunes y opciones metodológicas más pertinentes. 


\section{Perfil docente del profesor de la escuela rural}

El contexto escolar rural y las situaciones específicas que de este se derivan requieren una formación inicial particular de los docentes. Sin embargo, se percibe una falta de formación didáctico-curricular, de carácter específico, en el profesorado, característica en este tipo de centros, que suelen ser un paso obligado para los docentes en sus primeros años de docencia, circunstancia que se traduce en una escasa experiencia sobre la realidad educativa propia de la escuela rural (Boix, 2004 y 2011; Bustos, 2008 y 2011). Solo un reducido porcentaje del profesorado permanece en este tipo de centros de modo prolongado o indefinido.

La ausencia de una formación adecuada del profesorado, que responda a las necesidades que plantea la escuela rural ha sido recogida en diversos estudios, entre los que se referencian Bustos (2007) o el llevado a cabo por Hinojo, Raso e Hinojo (2010). Según estos estudios, ni la Universidad ni las administraciones educativas contemplan la formación inicial, ni continua del profesorado, necesarias para desarrollar métodos de enseñanza y aprendizaje en aulas con multigraduación, agrupamiento característico de la escuela rural, dependiendo casi exclusivamente de la buena voluntad de los profesores y las acciones compartidas entre ellos (Bustos, 2011). Lamentablemente, la Administración educativa también se ha hecho eco de que estas escuelas, generalmente, solo son lugares de paso para los profesores noveles, asumiendo el discurso de provisionalidad, sobre las mismas.

La situación no se da en países como Francia, donde sí hay una formación profesional y pedagógica específica para los profesores que van a llevar a cabo su labor docente en contextos rurales. Autores como Bustos (2011) señalan que esta búsqueda de formación específica en el ámbito referenciado surgió tras la detección de las carencias que mostraba el sistema educativo en este contexto. A raíz de lo cual, surgió L'Observatoire de l'Ecole Rurale, creado en 1999 por un grupo multidisciplinar de educadores, sociólogos, y geógrafos elementales. Los objetivos fundamentales que se proponían en el momento de su fundación se dirigían al desarrollo de métodos orientados, tanto a la formación inicial, como continua de los docentes. Iniciativa que facilitó la conexión con el medio rural y dio lugar a una movilidad nacional entre diferentes universidades.

La proyección del movimiento referenciado no solo tuvo incidencia en el ámbito universitario, sino también en las administraciones regionales y locales, sobre todo en aspectos relativos a conservación, sociedad y cultura rurales. Pero no solo Francia ha desarrollado una formación específica, Finlandia también posee un currículum de formación para los maestros noveles en los que se desarrollan programas permanentes que atienden a las peculiaridades situacionales de estos grupos, ya que la dispersión poblacional de este país y la existencia de poblaciones con censos muy bajos, demandan una atención singularizada para este tipo de centros. También en Inglaterra se muestra interés por esta modalidad formativa, que Hargreaves (2009) plantea como necesaria, atendiendo a las características demográficas y al contexto.

Volviendo al ámbito nacional, se constata como tras la revisión de la estructura curricular de los planos de estudios de los títulos de grado de Maestro, no se han contemplado la singularidad de este tipo de escuelas, mediante una mención específica, que adecuase la formación de los futuros egresados, para que puedan atender con garantías la formación de este tipo de alumnos (Hamodi y Aragués-Garde, 2014). Gran parte de la bibliografía consultada contempla que una proporción considerable del profesorado que desarrolla su ejercicio docente en centros de esta modalidad no supera los 4 años de experiencia profesional, razón por la que sería oportuno, ya en la formación permanente de los primeros años, trabajar con métodos para procesar la información; personales y de interacción social, que faciliten el desarrollo de la integralidad de la persona (Boylan, 2003). Pero ello, con ser necesario, no es suficiente. También se demanda la praxis personal de los maestros, que deben contribuir con iniciativas, trabajo colaborativo y motivación para neutralizar, en parte, sus carencias formativas (Ramírez-González, 2015).

Se considera desde esta colaboración la necesaria interconexión que debe existir entre el docente, 
el medio y la incorporación de las culturas del ámbito rural a los procesos formativos. Para conseguirlo, el docente debe hacer una exhaustiva programación de todas las actividades, tareas y contenidos, a fin de dar respuesta a las necesidades formativas de todos y cada uno de sus alumnos (Bustos, 2007). Tanto en su diseño como aplicación, el profesional de la educación debe tratar de establecer vías de comunicación con el entorno que constituye el contexto de los procesos de enseñanza y aprendizaje. Esta aproximación al ámbito rural, favorecerá el desarrollo de aprendizajes más significativos para el alumnado y la generación de vínculos entre la institución educativa, la comunidad y la cultura del lugar (Varela-Crespo, Varela-Garrote y Lorenzo-Castiñeiras, 2016).

La mediación de las diferentes realidades culturales de este tipo de centros, generará procesos biunívocos, ya que la escuela como el docente pueden ejercer una influencia positiva sobre la comunidad escolar en su conjunto, que puede beneficiar la generación de una cultura participativa (García-Prieto y Pozuelos-Estrada, 2017) y, por ende, a las decisiones a tomar ante los acontecimientos que suceden en el entorno. Pero, simultáneamente, el contexto también genera referentes para la orientación de la acción educativa. Todo ello, basado en la colaboración entre el centro educativo y la población de su entorno. Esta estrecha colaboración va a traducirse en grandes oportunidades para el aprendizaje y la definición de unas condiciones óptimas que favorezcan y compensen su desarrollo y, en cierto modo, permitan abordar la complejidad que supone hacer frente a grupos multigrado.

Las reflexiones anteriores vienen avaladas por la necesidad de "la calidad de las relaciones humanas y el clima de interacción interpersonal en los centros de enseñanza" (Raso, Marín y Rodríguez-García, 2017, 29). Es decir, la capacidad de trabajar colaborativamente del profesorado, de empatizar con las familias e implicarlas en la educación de sus hijos, sin obviar la importancia que tiene el desarrollo de la identidad profesional docente de los maestros y maestras para el desarrollo de los procesos formativos.

Profundizando sobre el perfil profesional de los docentes que suelen pasar por los CPRs, encontramos que, pese a la escasa formación de los maestros noveles, los grupos multigrado son el primer destino del profesorado, aspecto que debieran sopesar las diversas administraciones educativas, ya que comporta una proporción inversa entre preparación profesional y dificultad en el desempeño (Boix y Bustos, 2014) En cuanto a la permanencia del profesorado en este tipo de centros, se ha observado que no suele ser prolongada, cambiando de destino en cuanto tienen la oportunidad (Castillo, 2016). Sin embargo, algunas investigaciones (Bustos, 2007 y 2008) subrayan que la falta de experiencia del docente con grupos multigrado (Bustos, 2010) se equilibra con el paso del tiempo. Así, las actitudes negativas del profesorado en su etapa de inserción en gruposclase de escuela rural, se atenúan o se mantienen las positivas. Manifestaciones de frustración, ansiedad, desconcierto, sobreesfuerzo, resignación o ganas de marcharse a otros centros son valoradas con mayor incidencia en la etapa inicial de trabajo docente en condiciones de multigraduación y disminuyen con el paso del tiempo. Sin embargo, la satisfacción y la alegría en el trabajo aumentan con la experiencia docente en estos grupos. En este tipo de centros se considera de gran importancia: “Las 'agrupaciones flexibles' en la organización del estudiantado, especialmente en el aula multigrado o unidocente, en términos de que los agrupamientos no se conformen necesariamente por el nivel o edad cronológica correspondiente a cada estudiante, sino por las capacidades de cada quien." (Ramírez-González, 2015, 8).

Aspectos que se concretan en la organización del aula, en el desarrollo de la programación, en el modo de llevar a cabo el trabajo cooperativo o el autoaprendizaje, entre otros aspectos.

Gran parte de las dificultades que se originan al inicio de la actividad docente en estos grupos pueden estar relacionadas con un inadecuado modelo de formación inicial y de prácticas de enseñanza, ya que permiten que el profesorado llegue a los escenarios de la multigraduación sin la formación didáctica específica, necesaria para hacer frente a las exigencias que demanda el desempeño de su actividad docente (Heredero, Collado-González y Shoiti-Nozu, 2016), pero, sobre todo, con creencias pedagógicas poco favorables hacia ese tipo de centros. La experiencia docente en estos grupos se convierte con el paso del tiempo en el aspecto que invierte las actitudes negativas 
en positivas, más que el conocimiento previo. Este hecho sugiere también la necesidad de incentivar la continuidad en los centros con el objeto de rentabilizar el capital didáctico que acumula el profesorado tras un proceso de acomodación.

En un estudio presentado por Pérez-Ferra, Quijano, Ocaña y Martos se plantea que: "Los discursos de los docentes se tornan centrales en la investigación educativa, como manifestación de sus propias identidades y experiencias" $(2014,1)$. De modo que las creencias y significados que otorgan a su proceso de formación inicial, constituyen parte activa de su desarrollo como profesionales, en una constante retroalimentación con el contexto en el que desarrollan su actividad profesional. Y en este proceso se pueden generar y consolidar creencias positivas hacia este tipo de centros, fundamentalmente en la formación inicial.

Atendiendo a las preferencias del profesorado por trabajar en grupos multigrado o unigrado, son mayoría los que prefieren trabajar en grupos unigrado (próximo a las dos terceras partes) y solo una pequeña parte prefiere trabajar en grupos multigrado.

En el estudio de las posibles relaciones significativas entre variables, se constata que hay relación positiva entre el hecho de haber trabajado en grupos multigrado o unigrado y la preferencia de los mismos. El análisis establece que con el paso del tiempo disminuye la preferencia por trabajar en grupos de un solo grado (el profesorado que más cursos lleva impartiendo docencia en escuelas rurales opta en menor medida por esta preferencia), aumenta levemente la preferencia por grupos multigrado y también resulta mayormente indiferente trabajar en unos u otros para el profesorado que más cursos lleva en grupos multigrado.

Se constata que la motivación para la práctica docente en este tipo de contextos es escasa, ya que no conlleva incentivos económicos; no favorece la promoción de profesionales y requiere una inversión de tiempo mayor, pues no se dispone de recursos y materiales específicos para desarrollar la labor docente. En cambio, esa motivación puede proceder de otras fuentes como son la calidad de las relaciones interpersonales con el alumnado, su contexto de procedencia (familias, comunidad, etc.), entre el propio profesorado y la amplia posibilidad de desarrollar distintas áreas curriculares de manera transversal.

\section{Tipos de agrupamientos}

El profesorado debe diseñar y desarrollar el currículum de forma que defina las mejores condiciones para que su alumnado aprenda. En este sentido, gestionar adecuadamente la selección del tipo de agrupamiento, así como su imposición "obligada", adquiere gran importancia en su labor profesional. Basándose en su experiencia, el grupo de alumnos y las tareas a realizar, el docente optará por un agrupamiento mixto, individual, grupal, etc. Los maestros que trabajan en estos ámbitos no tienen como referente principal criterios de rendimiento en el aula, sino que intentan armonizar en los grupos modelos de agrupamiento flexibles, en los que se consideren todas las circunstancias, focalizando la justificación en la interacción entre los estudiantes (Bustos, 2007).

Los perfiles organizativos que surgen de los grupos multigrado suelen definir un contexto escolar en el que las metodologías a utilizar no se corresponden a agrupamientos definidos por curso-grado; al contrario, se plantean procesos de instrucción atendiendo a las necesidades generadas por el desarrollo madurativo del alumnado (Alvarado, 2010), superando una adscripción cronológica.

La adaptación de los procesos de enseñanza y aprendizaje a la realidad de los grupos de alumnos que se pueden definir en un centro rural, supone el inicio de una estrategia motivadora del profesorado respecto a los aprendizajes que han de adquirir los estudiantes (Block, Ramírez y Reséndiz, 2015). Dicho lo cual, se pasa a profundizar en el tipo de agrupamiento por el que se caracteriza la escuela rural, la multigraduación (Bustos, 2010). A lo largo de los años se han ido desarrollando unas concepciones que han dado sentido a la escuela rural y aún hoy siguen en un proceso de cambio para intentar suplir las distintas carencias que se plantean. Pero encontramos un 
aspecto que no ha sufrido cambio alguno a lo largo de estos años y no es otro que el que nos ocupa: la multigraduación. Esta forma de agrupamiento se presenta como la principal peculiaridad de la escuela rural (Boix y Bustos, 2014) y puede definirse como aquel tipo de agrupamiento en el que el alumnado de diferentes cursos, ciclos o etapas comparten las mismas situaciones de enseñanzaaprendizaje en una única aula.

Como consecuencia de la evolución que ha sufrido la escuela rural, el término multigraduación también se ha visto inmerso en este proceso de cambio. Sin embargo, ha sido la terminología y no su esencia significativa la que ha experimentado dicho cambio. Así, encontramos distintas denominaciones como multinivel, internivel, niveles compartidos, multiedad o multicurso, pero ninguno de estos términos se adecúa a su fundamento y significado. Aun siendo muy aceptada por el profesorado la denominación de "multiniveralidad", no se corresponde fielmente con la esencia de este tipo de agrupamiento escolar, ya que la existencia de diferentes niveles de aprendizaje está implícita en cualquier grupo de alumnos, por el principio de diversidad.

Considerando las reflexiones anteriores, tanto por las ideas que las sustentan como por la operatividad de las mismas, la multigraduación se asemeja más a la graduación multiedad (Hoffman, 2002). Ambas son una alternativa al sistema de grados y se alejan de los agrupamientos flexibles que nacen a partir de la Escuela sin grados, propia de los años sesenta y setenta (Miller, 1976). Mientras que la multigraduación depende de las circunstancias cuantitativas del alumnado, los agrupamientos flexibles parten de las decisiones docentes sin presentar un carácter administrativo. Pese a sus diferencias, ambas pueden coexistir pues los ambientes con multigraduación facilitan la realización de grupos flexibles (Bustos, 2010).

Otro aspecto que se ve favorecido por el modelo de agrupamiento multigrado es la monitorización entre alumnado, que estimula el trabajo cooperativo y la ayuda entre iguales a través del aprovechamiento de la heterogeneidad existente de edades y capacidades. Este fenómeno tiene como referente histórico la Escuela Mutua de Lancaster y Bell (Querrien, 2005).

En definitiva, no se puede hablar de escuela rural sin hacer alusión a la multigraduación, ya que esta última se desarrolla conforme a las concepciones organizativas que plantee la primera.

\section{Tipos de agrupamientos}

La agrupación, además de fortalecer los lazos afectivo-sociales entre los distintos agentes educativos, favorece los procesos de enseñanza y aprendizaje (Bustos, 2007).

Numerosos estudios realizados en el ámbito internacional han encontrado que los centros pequeños y rurales obtienen resultados académicos ligeramente superiores respecto a los centros grandes ubicados en núcleos urbanos (Núñez, Peña, Cubillos y Solorza, 2016; Uttech,2001). Además de los beneficios en cuanto a rendimiento, hallamos que las características propias de este tipo de centros fortalecen el componente afectivo-social (Bustos, 2011; Varela-Crespo, VarelaGarrote y Lorenzo-Castiñeiras, 2016). Entre ellos, podemos destacar el espíritu cooperativo que emerge en los primeros, las posibilidades de ofrecer una enseñanza individualizada, donde priman estrategias más significativas e innovadoras (García-Prieto y Pozuelos-Estrada, 2017), el enriquecimiento que viene intrínseco a la heterogeneidad que las escuelas multigrado ofrece, etc. (Block, Ramírez y Reséndiz, 2015).

En cuanto a los beneficios que reporta los agrupamientos multinivel al alumnado, los estudiantes de menor edad tienden a imitar y reproducir lo que hacen los mayores, siendo éstos últimos guía y apoyo. A través de prácticas colaborativas, los estudiantes de cursos superiores desarrollan el sentido de la responsabilidad (tanto individual como grupal), al tiempo que afianzan los conocimientos ya aprendidos. Además, se ejerce una colaboración y entendimiento mutuos, donde las rencillas y conflictos que puedan existir, son solventados mediante el trabajo cooperativo (Hamodi y Aragués Garde, 2014). A su vez, dado el reducido número de alumnos, es posible ofrecer una enseñanza individualizada (Smit y Humpert, 2012). 
Otro aspecto significativo facilitador de aprendizajes, es el uso de recursos del entorno rural en la organización de la clase. La cercanía al medio, junto al escaso número de alumnos, facilita la realización de actividades extraescolares, e incluso la impartición de las clases fuera del aula ordinaria, fortaleciéndose la relación alumnado-medio natural. Sin embargo, la mayor parte de investigaciones realizadas respecto a la Escuela Rural, han partido de un diagnóstico negativo, que ponía el acento sobre sus carencias, dotaciones de recursos, incorporación a la era tecnológica o formación de su profesorado, dejando al descubierto aquellos elementos que intervienen en el ambiente de aprendizaje del aula rural (Bustos, 2010; Vázquez-Recio, 2016).

\section{Conclusiones}

La revisión realizada muestra las necesidades formativas del profesorado que trabaja en contextos rurales. Es preciso que conozcan la escuela rural, crean en ella y la consideren como una escuela abierta, dinamizadora, plural, integradora y generadora de una nueva cultura, al mismo tiempo que se justifican los buenos resultados que suelen alcanzarse en este tipo de centros (Vázquez-Recio, 2016).

También es necesaria una actualización de la regulación legislativa de este tipo de centros, pues la norma vigente refleja escasos avances en cuanto a las escuelas rurales.

El mayor rendimiento que se constata en las escuelas multigrado, tiene entre sus posible razones, la elevada atención individualizada, facilitada por la baja ratio escolar que existe en estos grupos, que facilita mayor personalización de la enseñanza (Smit y Humpert, 2012), realidad que favorece el desarrollo de capacidades, el beneficio del alumnado, respecto a un aprendizaje "contagiado", ya que está inmerso en un escenario en el que se adelantan y se repasan constantemente contenidos de aprendizaje, debido a la presencia de alumnado de mayor y de menor edad.

De hecho, los alumnos de menor edad se familiarizan y conocen anticipadamente los contenidos correspondientes a próximos cursos escolares, a través del trabajo escolar del alumnado mayor y las explicaciones o demostraciones. Más allá de los aspectos académicos, se fortalecen otros factores igualmente importantes para la educación de los ciudadanos del mañana, como pueden ser la creación de vínculos afectivo-sociales; una cultura de colaboración entre iguales o el fomento de valores como el respeto mutuo y hacia el medio que les rodea o la empatía (Block, Ramírez y Reséndiz, 2015; Varela-Crespo, Varela-Garrote y Lorenzo-Castiñeiras, 2016). Posiblemente sean estos aspectos los que deberían servir de ejemplo para el resto de tipos de escuelas, claro que considerando la idiosincrasia de cada contexto. Incidir sobre ellos, en las dinámicas y culturas que les acompañan y sobre las que se construyen puede convertirse en una línea de investigación a continuar para alcanzar la mejora escolar tan buscada y diseñar e implantar un sistema educativo de calidad que recoja la voz de todos los agentes educativos, ofrezca igualdad de oportunidades y, sobre todo, responda a las demandas y necesidades de los protagonistas del proceso de enseñanza y aprendizaje (Alvarado, 2010).

Dicho todo lo cual, como una declaración de intenciones de deseos a cumplir, no hay que obviar la realidad de los hechos, verificados en la revisión de la literatura realizada. Tal vez el medio rural no sea una prioridad actual de los gestores en educación. El neopositivismo ha generado intereses vinculados a otros tipos de sociedades y el medio rural queda en una situación de tangencialidad.

Ante esta nueva realidad se constata que la formación inicial no considera, ni en el ámbito organizativo ni en el didáctico-curricular, la singularidad de estos contextos. Tampoco en la realización de las prácticas, los estudiantes solicitan de modo habitual centros de esta naturaleza. Recientemente, Pérez-Ferra y Quijano (2017) aluden a una percepción del practicum por los estudiantes que lo cursan, vinculada a una profesionalización, entendida como mero conocimiento de métodos. El sentido ético, lo orientan al compromiso del conocimiento de los mismos y a las corrientes sociales del momento. Situación problemática, ya que la incardinación del sentido ético deja de ser un sentir compartido, en este caso, por una comunidad profesional, surgida de la concepción moral compartida por todos. Esta realidad desvincula la ética profesional de su razón 
de ser en la educación, que Ortega-Ruiz ha definido del siguiente modo: "Mi propuesta es que la relación más radical y originaria que se establece entre maestro y alumno, en una situación educativa, es una relación ética que se traduce en una actitud de acogida y un compromiso con el educando, es decir, hacerse cargo de él” $(2005,5)$.

La propuesta que hace Ortega-Ruiz (2004) define la centralidad del hecho educativo; de lo que es vital para resolver el problema de profesionalización, tanto en las escuelas rurales como de otro tipo. Ser maestro no solo queda definido por conocer aspectos metodológicos y cómo gestionar el aula con los mismos, evidentemente, son necesarios, pero no se puede limitar la formación a mera instrucción.

Plantearse la profesión docente en las escuelas rurales, con un sentido ético, exige al profesorado orientar su identidad profesional hacia las necesidades de su alumnado. Querer ser docente tiene unas exigencias: ser profesional, ser reflexivo y tener sentido ético. Esa realidad es la que mueve las acciones didácticas, determinadas por los elementos didáctico-curriculares y por los condicionantes de la acción formativa: alumnos y clima de aula y, en tercer lugar, el "compromiso"; es decir, querer ser docente, el compromiso con los alumnos y la profesión (Pérez-García, 2015).

Se ha de trabajar con metodologías activas en este tipo de escuelas, pero no con una finalidad en sí misma. No se puede caer nuevamente en los mismos errores con los que se gestiona el gran legado de la Modernidad, la "acción"; en el hacer con finalidad en sí mismo y sin pretensiones teleológicas más allá de la verificación de hipótesis experimentalmente. Lo que sucede ya en la sociedad, instalada en un mero positivismo en el que se contrapone "competitividad" a "equidad", que es una de las razones por las que no se adecúa el hecho educativo a la realidad de los entornos rurales.

Sin duda, el nivel de racionalidad y optimización de la acción educativa ha mejorado con una educación más vinculada al sentido común y a la experiencia. Pero esta preocupación por la efectividad y el control de los aprendizajes deja a un lado la "integridad" de ser humano, determinante de la "integralidad" de los elementos constitutivos de la persona, que son los aspectos orientativos de la vida, que definen el avance de las sociedades.

\section{Referencias}

Alvarado, B. (2010). Voices and agencialities in the education of young rural Andean women: A qualitative look. Revista Electrónica de Investigación Educativa, 12(2), pp. 1-15. Recuperado de: http://redie.uabc.mx/vol12no2/contents-alvarado.html.

Bernal-Agudo, J. (2009). Luces y sombras en la escuela rural. Zaragoza: Unizar. Recuperado de: https://goo.gl/RUQ4Pr.

Block, D., Ramírez, M., \& Reséndiz, L. (2015). Las ayudas personalizadas como recurso de enseñanza de las matemáticas en un aula multigrado: un estudio de caso. Revista mexicana de investigación educativa, 20(66), pp. 711-735.

Boix, R. (2004). La Escuela Rural: Funcionamiento y Necesidades. Barcelona: Praxis.

Boix, R. (2011). ¿Qué queda de la escuela rural? Algunas reflexiones sobre la realidad pedagógica del aula multigrado. Profesorado. Revista de Currículum y Formación de Profesorado, 15(2), pp. 13-23. Recuperado de: https://recyt.fecyt.es/index.php/profesorado/article/view/43412/25285.

Boix, R., \& Bustos, A. (2014). La enseñanza en las aulas multigrado: Una aproximación a las actividades escolares y los recursos didácticos desde la perspectiva del profesorado. Revista Iberoamericana de Evaluación Educativa, 7(3), pp. 29-43. Recuperado de: https://revistas.uam.es/index.php/riee/article/view/3100/3299.

Boylan, C.R. (2003). Putting Rural Into Pre -Service Teacher Education. Australian Association for Research in Education. Recuperado de: https://www.aare.edu.au/publicationsdatabase.php/3722/Putting-rural-into-pre--service-teacher-education.

Bustos, A. (2007). Dos décadas de colegios públicos rurales. Una mirada a la escuela rural andaluza. Aula Abierta, 35(1-2), pp. 91-104. Recuperado de: https://goo.gl/9dnCLX.

Bustos, A. (2008). Docentes de escuela rural. Análisis de su formación y sus actitudes a través de un 
estudio cuantitativo en Andalucía. Revista de Investigación Educativa, 26(2), pp. 485-519. Recuperado de: http://revistas.um.es/rie/article/view/94041/90651.

Bustos, A. (2010). Aproximación a las aulas de escuela rural: heterogeneidad y aprendizaje en los grupos multigrado. Revista de Educación, 352, pp. 353-378.

Bustos, A. (2011). Investigación y escuela rural: ¿irreconciliables? Profesorado. Revista de Curriculum y Formación de Profesorado, 15(2), 155-170. Recuperado de: https://recyt.fecyt.es/index.php/profesorado/article/view/43498/25373.

Cantón, I. (2004). Intervención Educativa en la Sociedad del Conocimiento. Granada: Grupo Editorial Universitario.

Castillo, F.J. (2016). La escuela en el medio rural CRA "Ínsula Barataria". Repositorio Documental de la Universidad de Valladolid. Recuperado de: http://uvadoc.uva.es/handle/10324/18581.

Consejería de Educación y Ciencia, Junta de Andalucía (1988). Decreto 29/1988, de 10 de febrero sobre constitución de Colegios Públicos Rurales en la Comunidad Autónoma Andaluza (BOJA $\mathrm{n}^{\circ} 19$ de 4 de marzo de 1988). Recuperado de: http://www.juntadeandalucia.es/boja/1988/19/3.

Consejería de Educación y Ciencia, Junta de Andalucía (2017). Resolución de 12 de septiembre de la Dirección General de Profesorado y Gestión de Recursos Humanos, por la que se determina el desarrollo de las líneas estratégicas de formación del profesorado establecidas (BOJA, de 22 de septiembre). Recuperado de: http://www.juntadeandalucia.es/boja/2017/183/41.

Corchón, E. (2000). La escuela rural: pasado, presente y perspectivas de futuro. Barcelona: Oikos-Tau.

Elboj, C., Gil, A., Rodrigo, I., \& Zaldívar, I. (2004). La transformación de los Centros Rurales. Networks: An on-line Journal for Teachers Research, 7(1). doi: 10.4148/2470-6353.1162.

García-Prieto, F., \& Pozuelos-Estrada, F. (2017). El currículum integrado: los proyectos de trabajo como propuesta global para una escuela rural alternativa. Aula Abierta, 45(1), pp. 7-14. doi: $10.17811 /$ rifie.45.2017.7-14

Hamodi, C., \& Aragués-Garde, S. (2014). La escuela rural: ventajas, inconvenientes y reflexiones sobre sus falsos mitos. Palobra, 14, pp. 46-61. Recuperado de: http://ojs.udc.edu.co/index.php/palobra/article/view/631.

Hargreaves, L. M. (2009). Respect and responsibility: Review of research on small rural schools in England. International Journal of Educational Research, 48(2), pp. 117-128. doi: 10.1016/j.ijer.2009.02.004.

Heredero, E., Collado-González, I., \& Shoiti-Nozu, W. (2016). Los colegios rurales agrupados en España. Analisis del funcionamiento y organización de la escuela rural española a partir de un estudio de casos. Educação e Fronteiras On-Line, 4(12), pp. 142-153. doi: 10.30612/eduf.v4i12.4716.

Hinojo, F.J., Raso, F., \& Hinojo, M.A. (2010). Análisis de la organización de la escuela rural en Andalucía: problemática y propuestas para un desarrollo de calidad. Revista Iberoamericana sobre Calidad, Eficacia y Cambio en Educación, 8(1), pp. 80-105. Recuperado de: https://revistas.uam.es/index.php/reice/article/view/5376/5815.

Hoffman, J. (2002). Flexible grouping strategies in the multiage classroom. Theory into Practice, 41, pp. 47-52. doi: 10.1207/s15430421 tip4101_8.

Miller, R.I. (1976). La escuela no graduada. Una nueva solución educativa. Buenos Aires: El Ateneo.

Ministerio de Educación (2010). Real Decreto 132/2010, de 12 de febrero, por el que se establecen los requisitos mínimos de los centros que impartan las enseñanzas del segundo ciclo de la educación infantil, la educación primaria y la educación secundaria (B.O.E. de 12 de marzo). Recuperado de: http://www.anpeclm.com/web/attachments/article/1502/ Requisitos $\% 20$ minimos $\% 20$ de $\% 20$ Centros $\% 20$ Escolares.pdf.

Moyano, E. (2002). La sociedad rural en Andalucía. Jornada Autonómica de la Comunidad de Andalucía. Libro blanco de la agricultura y el desarrollo rural.

Núñez, C. G., Peña, M., Cubillos, F., \& Solorza, H. (2016). Estamos todos juntos: el cierre de la Escuela Rural desde la perspectiva de los niños. Educaşão e Pesquisa, 42(4), pp. 953-967. doi: $10.1590 / \mathrm{s} 1517-9702201612152334$.

Ortega-Ruiz, P. (2004). La Educación moral como Pedagogía de la Alteridad. Revista Española de Pedagogía, 62(227), pp. 5-30. Recuperado de: http://reunir.unir.net/handle/123456789/4130.

Pérez-Ferra, M., \& Quijano, R. (2017). El practicum: una experiencia que reconstruye o consolida las creencias pedagógicas de los futuros maestros. El practicum en la definición de la identidad 
profesional de los estudiantes de magisterio. Symposium Auto-Organizado, Poio, 5-7 de julio de 2017.

Pérez-Ferra, M., Quijano, R. Ocaña, M.T., \& Martos, J.M. (2014). Pedagogical beliefs of students of the teacher Degree in Early Childhood education training for professional practice. ECER 2015: Education and Transition. Budapest, 7 al 11 de septiembre. Recuperado de: http://www.eeraecer.de/ecer-programmes/conference/20/contribution/35610/.

Pérez-García, P. (2015). Querer ser docente. En Domingo, J., \& Pérez, M. (Coords.). Aprendiendo a enseñar. Manual práctico de Didáctica (pp. 69-74). Madrid. Pirámide.

Querrien, A. (2005). L'école mutuelle. Une Pédagogie trop efficace. Paris: Empêcheurs de Penser en Rond.

Ramírez-González, A. (2015). Valoración del perfil docente rural desde el proceso formativo y la práctica educativa. Revista Electrónica Educare, 19(3), pp. 86-111. doi: 10.15359/ree.19-3.9.

Raso, F., Marín, J.A., \& Rodríguez-García, A.M. (2017). Análisis de la satisfacción del profesorado de la escuela rural en la provincia de Granada (España) Respecto a su relación personal y profesional con la comunidad educativa. European Scientific Journal, 13(4), pp. 27-50. doi: 10.19044/esj.2017.v13n4p27.

Smit, R., \& Humpert, W. (2012). Differentiated instruction in small schools. Teaching and Teacher Education, 28(8), pp. 1152-1162. doi: 10.1016/j.tate.2012.07.003.

Urrutia, G., \& Bonfill, X. (2010). Declaración PRISMA: una propuesta para mejorar la publicación de revisiones sistemáticas y metaanálisis. Medicina Clínica, 135(11), pp. 507-511. doi: 10.1016/j.medcli.2010.01.015.

Uttech, M. (2001). Imaginar, facilitar, transformar. Una pedagogía para el salón multigrado y la escuela rural. Buenos Aires: Paidós Mexicana.

Varela-Crespo, L., Varela-Garrote, L., \& Lorenzo-Castiñeiras, J.J. (2016). La escuela y la conciliación de los tiempos cotidianos de las familias del alumnado de educación primaria en Galicia. Bordón. Revista de Pedagogía, 68(4), 179-194. doi: 10.13042/Bordon.2016.48602.

Vázquez-Recio, R. (2016). Las escuelas públicas rurales: entre el bien común y la exclusión. Revista Interuniversitaria de Formación del Profesorado, 30(1), 67-79. Recuperado de: http://www.aufop.com/aufop/revistas/arta/impresa/194/1997. 
